\title{
Numerical and experimental study of the fluid flow through a medical device
}

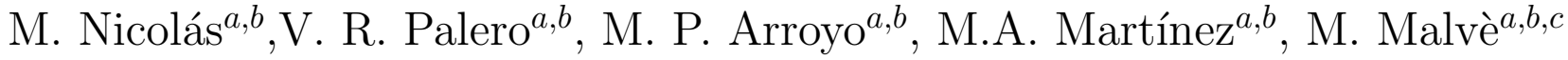 \\ ${ }^{a}$ Aragón Institute of Engineering Research (I3A). Universidad de Zaragoza. \\ C/María de Luna s/n, E-50018 Zaragoza, Spain \\ ${ }^{b}$ Centro de Investigación Biomédica en Red en Bioingeniería Biomateriales y \\ Nanomedicina (CIBER-BBN) C/Poeta Mariano Esquillor s/n, E-50018 Zaragoza \\ ${ }^{c}$ Public University of Navarra, Department of Mechanical Engineering, Energetics \\ and Materials, Pamplona, Spain \\ E-mail: marinanc@unizar.es
}

\begin{abstract}
The purpose of this paper is to verify a commercial software based fluid-structure interaction scheme for the inferior vena cava. Vena cava deep thrombosis (TVP) is a potentially deathly disease consequent to pulmonary thromboembolism (TEP). TEP consist in the obstruction of the pulmonary artery due to a blood clot traveling in the cardiovascular system and is treated with anticoagulants and inferior vena cava filters. Flow fields along the vena cava and an antithrombus filter were studied and compared with a Particle Image Velocimetry (PIV) based model to validate the numerical model. The results show that the Fluid Structure Interaction (FSI) models are valid and can be used to study the deformations in the inferior vena cava wall using patient-specific geometries.
\end{abstract}

Key words: Inferior Vena Cava, FSI, CFD, PIV, antithrombus filter 


\section{Introduction}

Pulmonary thrombotic embolism, known as PTE, is still a serious and frequent cardiovascular disease, despite the advances in surgical techniques and therapies. Cardiovascular diseases are one of the main causes of death in developed countries and are an important subject of interest in the biomedical engineering research field. PTE is a significant clinical problem worldwide. In the United States alone, there are estimated two million cases per year (1). Usually the main therapies used for the treatment of PTE are anticoagulation and fibrinolysis but sometimes they are contraindicated or ineffective, so it is necessary to use other techniques. Inferior vena cava filters have been shown to be effective in trapping embolized clots and preventing PTE (2). They were designed for the first time in 1967 and since then their design has been improved considerably and their use has increased (3). They have proved to be effective and secure treating PTE but it still remains unknown which type of filter is the best. Ideally, the filter should be effective while being nonthrombogenic and nonimpeding to the blood flow, and should have the ability to break the clot once captured. In reality, the filter has to establish a balance between clot capture efficiency and flow impedance before and after clot capture (1).

While there have been a number of detailed hemodynamic studies of the aorta and major arteries, far fewer have been done in the major veins, such as the inferior vena cava (IVC)(4). Previous experimental and computational studies have investigated the hemodynamic effects of IVC filter placement and embulus capture rate in simplified IVC geometries (5). Many in vitro studies have been performed using optical methods such as particle imagine velocimetry $(6 ; 7 ; 8 ; 9 ; 10)$. (11; 12;13) have studied flow fields surrounding a vena cava filter using a noninvasive technique called photochromic. $(14 ; 15 ; 16)$ validate numerical results with the esxperimental 2D velocity fields obtained with PIV (17).

A more complex study was developed by (2) where numerical models were created with different commercially available filters and different clot shapes, and an in vitro experiment was developed using Digital Particle Imaging Velocimetry Technique in 
order to compare the theoretical results obtained with the in-vitro experiments. Invitro experiments are effective to study the filter flow dynamics but they also have setup limitations that sometimes do not allow to create very realistic scenarios. Invitro experiments are an useful tool used to validate numerical models.

Numerical studies on vena cava filters are normally focused on hemodynamic aspects, neglecting the interaction between blood flow, venous walls and the filter. $(18 ; 19)$ evaluated the flow hemodynamics of the TrapEase and Celect vena cava filters using three dimensional computational fluid dynamics, including simulated thrombi of multiple shapes, sizes, and trapping positions. The study was performed to identify potential areas of recirculation. (1) developed a numerical model that characterized the hemodynamics of the flow around a Greenfield Vena Cava filter and that also wanted to determine the clot capturing efficacy.

The computation of fully coupled fluid flows with structural interactions is a rapidly evolving discipline that has been given increased attention in recent years (20). FSI analysis is very important when the structure undergoes large deformations resulting from the presence of a fluid. FSI studies in the inferior vena cava are very important, which to our knowledge have not been performed before. The inferior vena cava undergoes large deformations during Valsalva maneuver and the presence of a filter affects drastically the deformations that occur in the vena, so it is very important to start considering the IVC as a subject of FSI studies. Much has been accomplished in FSI modeling research since the early 1990s, and a good portion of that FSI research has been directed towards arterial fluid mechanics (21). There are a number of FSI finite element analysis in cardiovascular diseases such as arterial stenosis $(22 ; 23 ; 24)$, studies on left ventricular flow $(25)$ and others in abdominal aortic aneurism (26).

However, nothing can be found for inferior vena cava studies. (27) developed a computational rigid model of the inferior vena cava to study the hemodynamics of an unoccluded and partially occluded IVC and he stated that a computer model that accounts for the fluid-structure interaction between the flow and the vessel/thrombus would provide a more realistic model. An important feature of the 
inferior vena cava to study is its predisposition to undergo large displacements. The blood flow depends on the vena geometry, and the deformation of the vena depends on blood flow. For that reason, the equations governing the blood flow and vena deformation need to be solved simultaneously, with proper kinematic and dynamic conditions coupling the two physical systems (21). FSI analysis requires considerable computational power to run (28), so it important to investigate if its use is justified over CFD analysis.

In this study, an in-vitro model is used to verify a commercial software based fluid structure interaction scheme for the inferior vena cava. The velocity profiles of both numerical FSI and experimental PIV models are compared.

\section{Materials and Methods}

\subsection{Geometries and numerical grid generation}

Computer-aided design models were created using the commercial software SolidWorks (Dessault, SolidWorks Corp., France). There are three separate CAD models to be analyzed. First, a $12 \mathrm{~mm}$ diameter, $1.6 \mathrm{~mm}$ thick and $46 \mathrm{~mm}$ long idealized cylindrical vena cava was modelled. Second, a model that includes an antithrombus filter attached to the vena wall was designed. The filter was modelled by taking micrometer measurements to help as a guide. The angles between the filter wires were set so that the filter fits properly within $12 \mathrm{~mm}$ inner diameter vena cava. Finally, a third model that includes a clot trapped by the IVC filter was modelled. The clot was modelled as a semi circle and a cone joined and had a maximum diameter of $6 \mathrm{~mm}$. FSI simulations require more resources and time in terms of computational data acquisition (28). For that reason, a first simple model with only the idealized vena cava was done. Once the software was validated and the method used was of interest, the second model was analyzed.

Finally, using Ansys Icem (Ansys Inc. SOftware, Canonsburg, PA, USA) we ob- 
tained the numerical grid. Tetrahedral elements were used for the finite elements mesh using different mesh density depending on the part of the model. In the contact zones between the filter struts and the vein, the mesh density was greater as opposed to the wall of the vena where the elements density is small to accelerate the calculation. The geometry was divided into different parts in order to assign the desired element size to each part of the model. In order to simulate a fully developed flow, the mesh was extruded $20 \mathrm{~mm}$ before the inlet and after the outlet of the cylinder. In order to guarantee that the numerical results were grid independent a mesh independence study was carried out prior to the preseneted simulations. Velocity profiles at different vena cava locations were compared for different grid sizes. This comparison clearly demonstrated that the mesh finer that 1000000 elements increased the computational time without adding precision to the solution. Compared profiles differed in fact only less than $1 \%$.

The computational grids for the first empty vena model possessed finally 500000 elements for the solid part and 800000 elements for the fluid part. The second model which adds the filter had 1000000 elements in the fluid part and 600000 elements in the solid part (see Figure 1 for mesh details). The third model had 800000 elements in the fluid part and 800000 elements in the solid part.

The final meshes were imported into the software package ADINA Inc (Watertown, MA, U.S.A.) where the FSI simulations were performed.

\subsection{FSI Model properties and boundary conditions}

The final mesh was imported into the software package ADINA $R \& D$ Inc (Watertown, MA, U.S.A.). where the FSI simulations were performed. FSI was used in conjunction with the flow visualization studies to provide details on the effects of the filter and the clot on the flow field. A set of three filter/vena cava/clot models were created to match as closely as possible those used in the flow visualizations. The fluid used for the FSI model had the same properties as the fluid used in the ex- 
perimental model, which was a mixture of water and glycerol and had a newtonian behavior. Though blood is a non-Newtonian fluid, (1) showed that the Newtonian approximation introduces less than $10 \%$ error in velocities when simulating flow in a simplified IVC geometry (5). The density of the fluid was $1.07 \mathrm{~g} / \mathrm{cm}^{3}$ and its viscosity was $0.041 \mathrm{~g} / \mathrm{cms}$. Since the Reynold number based on the average vena diameter was $R e=145$, the fluid flow was assumed laminar and incompressible under steady conditions. Previous studies have found that transitional flow does not have a significant impact on the overall flow though the IVC, even when the IVC is partially occluded (27), so the laminar flow assumption can be considered well done. The vena wall was modelled to behave as silicon. The wall was assumed to be isotropic, with a Young's modulus of $1000 \mathrm{MPa}$, Poison ratio of 0.3 and to undergo large displacements. The filter was made of steel and had a Young Modulus of $210000 \mathrm{MPa}$ and a Poisson ratio of 0.3 . The clot was modeled with a Young's modulus of $1200 \mathrm{MPa}$ and a Poisson rati of 0.3 .

As usual for FSI analyses, mixed velocity-pressure condition are required in order to correctly compute flow features and structural stresses, strains and displacements. In this work uniform velocity and pressures measured in the experimental setup were used as the boundary conditions. Both velocity and pressure were constant and were applied progressively until it reached the desired value. The flow and pressure were applied at the inlet and outlet of the fluid domain respectively to match the experimental setup. The fluid flow was laminar and the pressure was applied as normal traction. No-slip wall boundary condition was imposed at the fluid-solid interface. Finally, at the extensions extremities of the solid model, no axial or transaxial motion was permitted. This constraint, though non-physiological, as the inlet and outlets should be allowed to deform radially to correctly simulate tethering of the artery, is commonly assumed in finite element studies of cardiovascular vessels (29).

To solve the coupling between the fluid and the solid models, the iterative method was chosen (? ). In this computing method, the fluid and solid solution variables are fully coupled. The fluid and solid equations are solved individually in sucession, 
always using the latest information provided from another part of the coupled system. This iteration is continued until convergence in the solution of the coupled equations is reached. The maximum number of fluid- structure iterations for each time step was set to 1000 . The time size was set to $0.001 \mathrm{~s}$.

Simulations were performed in parallel on one node of a cluster with an Intel processor, 8 CPUs, with a CPU speed of $2268 \mathrm{MHz}$ and a total memory of $24 \mathrm{~Gb}$.

It has to be noted that the calculation time for of the 3 FSI simulations was around $1080 \mathrm{~h}$.

2.3 Digital Imaging Velocimetry Technique (PIV)

Because the flow field surrounding an IVC filter is complex and three-dimensional, a detailed hemodynamic assessment presents a considerable technique challenge. Flow measurement techniques are limited and their ability to accurately characterize complex flow fields, especially near solid boundaries. Difficulties with optical techniques may be encountered (11). Particle image velocity has an important advantage over other optical techniques that are used for flow measurements. It produces two-dimensional or three-dimensional vector fields while other techniques such as laser Doppler velocimetry only measure the velocity at a point. For that reason PIV has been used in this study. Particle image velocimetry (PIV) is a well known technique for the measurement of the in-plane velocity components in a seeded fluid plane illuminated with a laser sheet. PIV records the position over time of small tracer particles introduced into the flow to extract the local fluid velocity (30). The analysis of consecutive recordings of the fluid plane, taken at known time intervals, provides the whole velocity field in such plane (31). 


\subsection{Experimental set-up}

The experimental set up was used to measure the flow field in the central plane of several vein models, using particle image velocimetry (PIV). 2 different models were analyzed. Firstly, flow was visualized in an idealized inferior vena cava model, set horizontally, made of $2 \mathrm{~mm}$ thick silicone with an internal diameter of $12 \mathrm{~mm}$. This model was used to validate the numerical software that was going to be used later to analyze more complex models. Secondly, a commercially available filter was added to the model to study its effect on the blood flow, velocity patterns, and wall shear stress by flow visualization and computational fluid dynamics(2). A four legs Gunther Tulip was deployed into the vena cava symmetrically in the center of the model. A High Speed two-cavity New Wave Pegasus laser $(\lambda=527 n m$, energy per pulse $=10 \mathrm{~mJ}$ at $1000 \mathrm{~Hz}$ ) was used as a light source. A high speed camera (Fastcam SA2, sensor size of $2048 \times 2048$ pixels, pixel size of $11 \mu m x 11 \mu m$ ), was used to record the PIV images. The camera was working at its maximum full size recording rate: $1000 \mathrm{images} / \mathrm{s}$. Each laser was fired at half this rate (500 pulses/s). Two identical lenses $(f=55 \mathrm{~mm}$ ) were used to image the illuminated fluid plane into both sensors with a magnification close to unity $(M=0.935)$. The lenses were working at $f=22$ (32). The fluid used to simulate the blood inside the veins was $40 \%$ by weight of glycerin and $60 \%$ by weight of water with viscosity $0.041 \mathrm{gcm}^{-1} \mathrm{~s}^{-1}$ and density $1.07 \mathrm{~g} \mathrm{~cm}^{-3}$. A pump was used to drive steady flow at $352 \mathrm{ml} / \mathrm{min}$. The PIV images were recorded at $1000 \mathrm{~Hz}$ and analyzed using the Davis software from LaVision. The option of PIV sum of correlation was selected. This feature is convenient when the fluid seeding is sparse, as the final displacement vector is calculated by adding the cross-correlation peak in the same interrogation window (iw) in the whole image series. The velocity map was obtained in two steps. In the first one the iw size was 64 pixels and the overlapping between consecutive ones was 50\%. In the second step the iw size was reduced to 32 pixels with a $50 \%$ of overlapping (32).

Although the flow patterns in the numerical models were acknowledged to be threedimensional, the PIV technique used was limited to determining the velocoties in a 
two-dimensional plane. Therefore particle paths were calculated in the midplane of the PIV studies, as well as in the FSI studies for comparison.

\section{Results}

A comparison between 2 different nuemerical and experimental models has been done. First, an idealized vena cava and then a model including the filter. Then, once the numerical software has been validated, a third numerical FSI model including a clot has been analyzed as an example of application of the FSI method.

The velocity along the system was analyzed first. For the empty vena model it was observed that the velocity profile did not vary as the flow moves along the vena, as it could be expected, and the maximum velocity was recorded to be $0.9 \mathrm{~m} / \mathrm{s}$. Figure 2c shows changes in though-plane velocities over a distance of $60 \mathrm{~mm}$ along the vessel axix. The slides are the same distance apart from each other in the three models analyzed. Notice that there is barely no variation in the velocity isocontours from slice to slice.

A velocity comparison was made between the FSI model and the experimental PIV model in order to validate the software ADINA and the numerical method used. Velocity data was taken at to different points along the symmetry plane of the vena and the velocity profiles of both numerical and experimental models were compared. Figure $2 \mathrm{a}$ and $\mathrm{b}$ show the velocity at two different points, the first one closer to the inlet and the second one closer to the outlet. It is observed that the velocity profiles for both numerical and experimental models are very similar to each other and the maximum values are also very alike. Both the FSI results and the PIV data showed the expected parabolic velocity profile, with largest velocity in the middle of the vena and zero velocity at the wall.

Once the first simplified model was validated and demonstrated to be efficient analyzing velocity fields, a second model including the Gunther Tulip filter was ana- 
lyzed. Figure 3 shows velocity contour plots of the axial velocity represented along the symmetry plane of both experimental and numerical models. Qualitative agreement between the flow visualization experiment and the FSI results was observed. Velocity profiles were consistent with results from previous studies $(12 ; 9 ; 6 ; 2)$.

As it can be seen in Figure 3, the in-plane velocity for both models is very similar. It is observed flow with zero velocity adjacent to all solid boundaries (i.e., vessel walls and filter), as expected from the no-slip boundary condition. The flow diverged around the wires of the filter and then converged on the downstream side. To have a quantitative understanding of the velocity along the model, the velocity profile has been represented at different points of the model. Figure ?? shows 5 points where the velocity has been studied. Line $A$ shows the velocity at the beginning of the model, a few millimeters after the inlet. Line $B$ is taken just at the end of the filter struts. Line $C$ is taken at approximately the mid-point of the filter, line $D$ is taken at the end of the filter, and goes right through the tip of the filter and finally line $E$ is taken after the filter, before the outlet. The velocity has a parabolic profile having a maximum value of $0.09 \mathrm{~m} / \mathrm{s}$ in the middle and minimum of $0 \mathrm{~m} / \mathrm{s}$ at points close to the wall. Since the velocity lines measured are in the symmetry plane of the model, the velocity profiles analyzed are going to have a value of 0 only when it passes through the tip of the filter, along line $D$ (as it is positioned in the center of the model). The struts of the filter are not on the symmetry plane, so for that reason the velocity profiles are not 0 on Figure ?? b, c and d which are along the filter. The velocity is zero at the wall and when the fluid flows though the filter. Once the fluid passes the filter it recovers its normal behaviour being maximum at the middle and minimum at the extremes. From Figure 4 it is observed how the maximum velocity is mainly the same along the entire model, as the flow advances though the vena. At point $\mathrm{A}, \mathrm{B}$, and $\mathrm{C}$ the maximum velocity is $0.09 \mathrm{~m} / \mathrm{s}$ Figure 5 shows the velocity isocontours at the same points that the velocity profiles have been plotted. 


\section{Disscussion}

A fluid-structure interaction (FSI) model for anaylizing the hemodinamics of a symplified IVC/antithrombus filter was developed. Fluid-structure interaction (FSI) techniques were introduced to increase the scope, accuracy of the vena cava model. Although the study of the wall deformations was not in the scope of this proyect, it is imporant to validate the ADINA software for FSI studies. The inferior vena cava wall undergoes enormous deformations so it is important to know that FSI analysis on realistic geometries can be done and the deformations can be studied. This studied has proved that the ADINA model works correctly, so the next step would be to study the deformations for patient-specific IVC geometries, with the filter inserted. As mentioned by (27), the rigid model assumptions may over-estimate the WSS and may be an upperbound on the true physiological value. It has to be noted that this work does not want to present a realisit study of an IVC but validate a FSI model by comparing it with an in-vitro model.

As mentioned by (2), who studied the blood flow for different filter models, patterns of flow past IVC filters were observed to be highly dependent on filter design. For that reason, this is study can be used as a reference only for umbrella type filters. As it usually happens in every study, this work is affected by some simplifications. This study was limited to steady flow in a circular caval model. The vena cava is compliant and elliptical, with pulsatile fluctuations altered by respiration. However, steady-flow analysis is widely viewed as a means of providing the time-averaged behavior of pulsatile flow conditions (9). Blood was considered to be newtonian but blood is known to behave in a non-Newtonian fashion particularly at low flow rates. In a vessel of this diameter these effects will not be significant (13). The simplifications of this simulation, such as thin-wall vena cava geometry, isotropic and homogeneous material and imposing uniform inflow velocity, obviously may limit the actual clinical uses. However, the model has validated the FSI software and can now be used for more realistic studies. Fluid and structure models can be both mechanically and thermally coupled along fluid-solid interfaces and also though 
shared regions. The accurate analysis of these coupled problems requires a powerful structural program, a powerful fluid program and a general coupling scheme (20).

\section{Conclusions}

A 3-D thin-wall model with fluid-structure interactions was introduced and solved using ADINA to investigate the blood flow in a vena cava wall with an antithrombus filter inserted. It was a preparation for further simulations concerning the real inferior vena cava. The PIV showed at some point some non-symmetry. This was likely due to the curvature of the vena suffered during testing, but for both models the comparison between the PIV model and the FSI model was good.

\section{Acknowledges}

The authors gratefully acknowledge research support from the Spanish Ministry of Science and Technology through the research project DPI2010-20746-C03-01, and the CIBER initiative. We also thank the Spanish Ministry for the financial support to M. Nicolas through the grant BES-2011-043471.

\section{References}

[1] T. N. Swaminathan, H. H. Hu, A. a. Patel, Numerical analysis of the hemodynamics and embolus capture of a greenfield vena cava filter., Journal of biomechanical engineering 128 (3) (2006) 360-70.

[2] S. F. C. Stewart, R. a. Robinson, R. a. Nelson, R. a. Malinauskas, Effects of thrombosed vena cava filters on blood flow: flow visualization and numerical modeling., Annals of biomedical engineering 36 (11) (2008) 1764-81.

[3] H. Lorch, A. Dallmann, M. Zwaan, H.-D. Weiss, Efficacy of permanent and re- 
trievable vena cava filters: experimental studies and evaluation of a new device., Cardiovascular and interventional radiology 25 (3) (2002) 193-9.

[4] C. P. Cheng, R. J. Herfkens, C. a. Taylor, Inferior vena caval hemodynamics quantified in vivo at rest and during cycling exercise using magnetic resonance imaging., American journal of physiology. Heart and circulatory physiology 284 (4) (2003) H1161-7.

[5] K. I. Aycock, R. L. Campbell, K. B. Manning, S. P. Sastry, S. M. Shontz, F. C. Lynch, B. a. Craven, A computational method for predicting inferior vena cava filter performance on a patient-specific basis., Journal of biomechanical engineering 136 (8) (2014) 1-13.

[6] A. Harlal, M. Ojha, K. W. Johnston, Vena cava filter performance based on hemodynamics and reported thrombosis and pulmonary embolism patterns, Journal of Vascular and Interventional Radiology 18 (1) (2007) 103-115.

[7] H. J. Jaeger, S. Kolb, T. Mair, M. Geller, A. Christmann, R. K. H. Kinne, K. D. Mathias, In vitro model for the evaluation of inferior vena cava filters: Effect of experimental parameters on thrombus-capturing efficacy of the Vena Tech-LGM filter, Journal of Vascular and Interventional Radiology 9 (1998) 295-304.

[8] A. A. Katsamouris, A. C. Waltman, A. Delichatsios, A. Athanasoulis, Inferior Vena Cava Filters : In vitro comparisn of Clot Trapping and Flow Dynamics, Cardiovascular radiology (166) (1988) 361-366.

[9] R. L. Leask, K. W. Johnston, M. Ojha, In vitro hemodynamic evaluation of a Simon nitinol vena cava filter: possible explanation of IVC occlusion, Journal of vascular and interventional radiology : JVIR 12 (5) (2001) 613-8.

[10] G. W. Stoneham, B. E. Burbridge, S. F. Millward, Temporary inferior vena cava filters: in vitro comparison with permanent IVC filters, Journal of Vascular and Interventional Radiology 6 (5) (1995) 731-736.

[11] G. G. Couch, K. Johnston, M. Ojha, An in vitro comparison of the hemodynamics of two inferior vena cava filters, Journal of Vascular Surgery 31 (3) (2000) 539-549. 
[12] R. L. Leask, K. W. Johnston, M. Ojha, Hemodynamic Effects of Clot Entrapment in the TrapEase Inferior Vena Cava Filter, Journal of Vascular and Interventional Radiology 15 (5) (2004) 485-490.

[13] G. G. Couch, H. Kim, M. Ojha, In vitro assessment of the hemodynamic effects of a partial occlusion in a vena cava filter., Journal of vascular surgery 25 (4) (1997) 663-72.

[14] Y. Hoi, S. H. Woodward, M. Kim, D. B. Taulbee, H. Meng, Validation of CFD simulations of cerebral aneurysms with implication of geometric variations., Journal of biomechanical engineering 128 (6) (2006) 844-51.

[15] G. Mulder, A. C. B. Bogaerds, P. Rongen, F. N. Vosse, On automated analysis of flow patterns in cerebral aneurysms based on vortex identification, Journal of Engineering Mathematics 64 (4) (2009) 391-401.

[16] M. D. Ford, H. N. Nikolov, J. S. Milner, S. P. Lownie, E. M. Demont, W. Kalata, F. Loth, D. W. Holdsworth, D. a. Steinman, PIV-measured versus CFDpredicted flow dynamics in anatomically realistic cerebral aneurysm models., Journal of biomechanical engineering 130 (2) (2008) 021015.

[17] V. R. Palero, J. Lobera, M. P. Arroyo, Three-component velocity field measurement in confined liquid flows with high-speed digital image plane holography, Experiments in Fluids 49 (2) (2010) 471-483.

[18] M. a. Singer, W. D. Henshaw, S. L. Wang, Computational modeling of blood flow in the TrapEase inferior vena cava filter., Journal of vascular and interventional radiology : JVIR 20 (6) (2009) 799-805.

[19] M. A. Singer, S. L. Wang, Modeling Flow Past a Tilted Vena Cava Filter, Journal of biomedical engineering submitted.

[20] H. Zhang, X. Zhang, S. Ji, Y. Guo, G. Ledezma, N. Elabbasi, H. DeCougny, Recent development of fluid structure interaction capabilities in the ADINA system, Computers \& Structures 81 (8-11) (2003) 1071-1085.

[21] T. E. Tezduyar, S. Sathe, T. Cragin, B. Nanna, B. S. Conklin, J. Pausewang, M. Schwaab, Modelling of fluid structure interactions with the space time finite elements : Arterial fluid mechanics, International Journal for Numerical 
Methods in Fluids (February) (2007) 901-922.

[22] D. Tang, C. Yang, S. Kobayashi, J. Zheng, R. P. Vito, Effect of Stenosis Asymmetry on Blood Flow and Artery Compression: A Three-Dimensional FluidStructure Interaction Model, Annals of Biomedical Engineering 31 (10) (2003) $1182-1193$.

[23] M. Bathe, R. D. Kamm, A fluid-structure interaction finite element analysis of pulsatile blood flow through a compliant stenotic artery., Journal of biomechanical engineering 121 (4) (1999) 361-9.

[24] D. Tang, C. Yang, D. N. Ku, A 3-D thin-wall model with fluid-structure interactions for blood flow in carotid arteries with symmetric and asymmetric stenoses 72 (1999) 357-377.

[25] Y. Cheng, H. Oertel, T. Schenkel, Fluid-Structure Coupled CFD Simulation of the Left Ventricular Flow During Filling Phase, Annals of Biomedical Engineering 33 (5) (2005) 567-576.

[26] C. M. Scotti, J. Jimenez, S. C. Muluk, E. a. Finol, Wall stress and flow dynamics in abdominal aortic aneurysms: finite element analysis vs. fluid-structure interaction., Computer methods in biomechanics and biomedical engineering 11 (3) (2008) 301-22.

[27] Z. Ren, S. L. Wang, M. A. Singer, Modeling hemodynamics in an unoccluded and partially occluded inferior vena cava under rest and exercise conditions, Medical \& Biological Engineering \& Computing (50) (2012) 277-787.

[28] J. H. Leung, A. R. Wright, N. Cheshire, J. Crane, S. a. Thom, A. D. Hughes, Y. Xu, Fluid structure interaction of patient specific abdominal aortic aneurysms: a comparison with solid stress models, Biomedical engineering online 5 (2006) 33 .

[29] M. Malvè, a. García, J. Ohayon, M. Martínez, Unsteady blood flow and mass transfer of a human left coronary artery bifurcation: FSI vs. CFD, International Communications in Heat and Mass Transfer 39 (6) (2012) 745-751.

[30] M. Jahanmiri, Particle Image Velocimetry : Fundamentals and Its Applications Particle Image Velocimetry : Fundamentals and Its Applications, Tech. 
rep., Division of fluid dynamics, Department of Applied Mechanics,Chalmers University of Technology, Göteborg, Sweden (2011).

[31] M. Raffel, C. E. Willert, S. Wereley, J. Kompenhans, Particle Image Velocimetry, a practical guide, 2nd Edition, Springer Verlag, 2007.

[32] V. R. Palero, E. M. Roche, L. Arévalo, M. Nicolás, M. Malvè, M. A. Martinez, M. P. Arroyo, in: 15th International Symposium on Flow Visualization, Minsk, Belarus, 2012. 

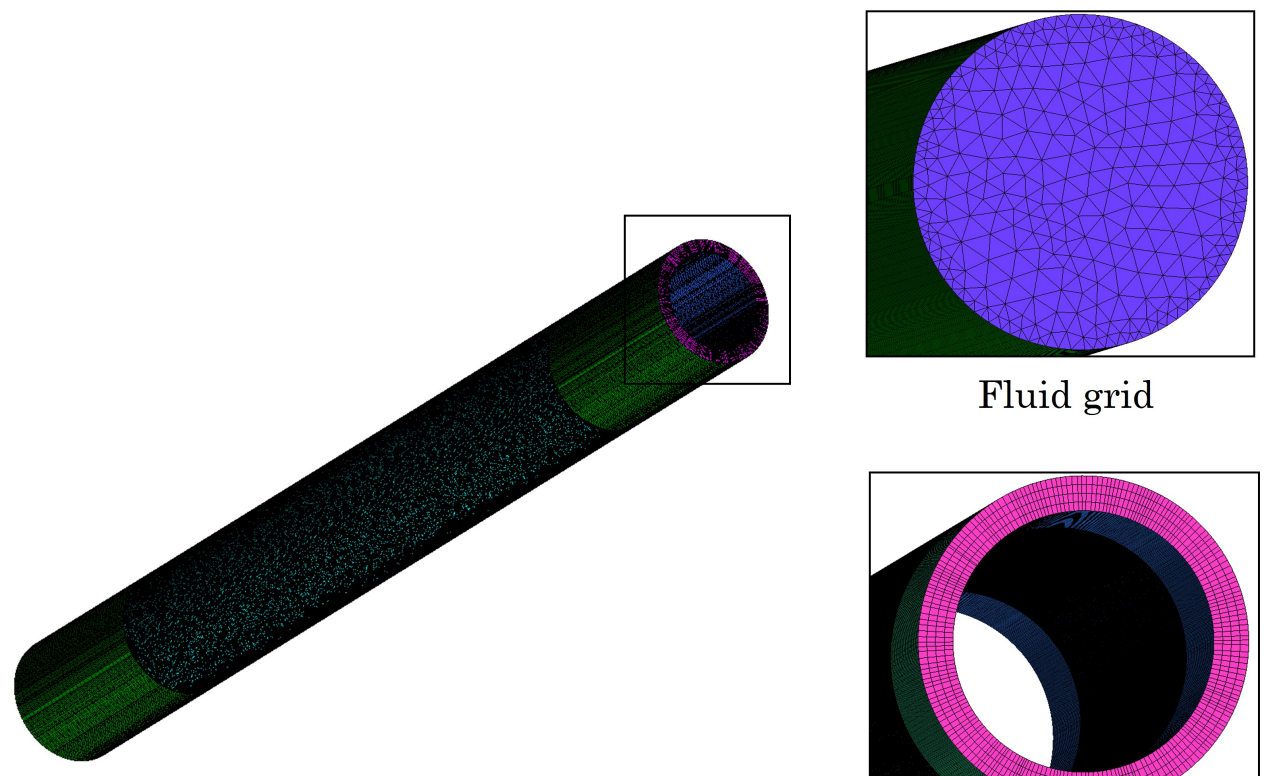

Fluid grid

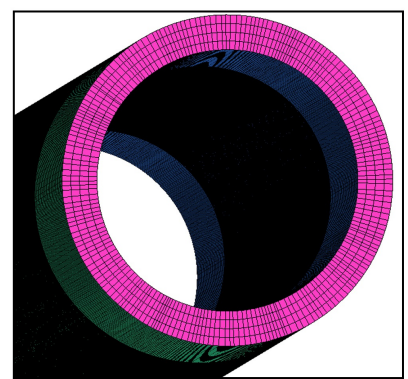

Solid grid

(a)

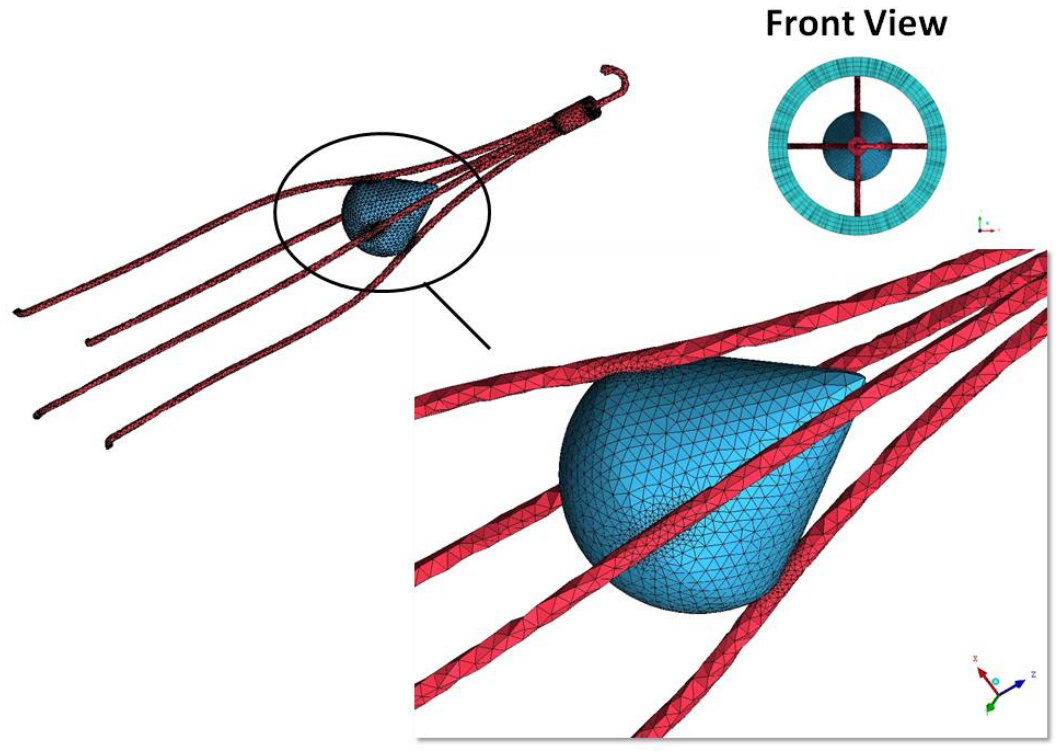

(b)

Fig. 1. a) Solid mesh for the emptly vena model, b) Mesh for the IVC filter 


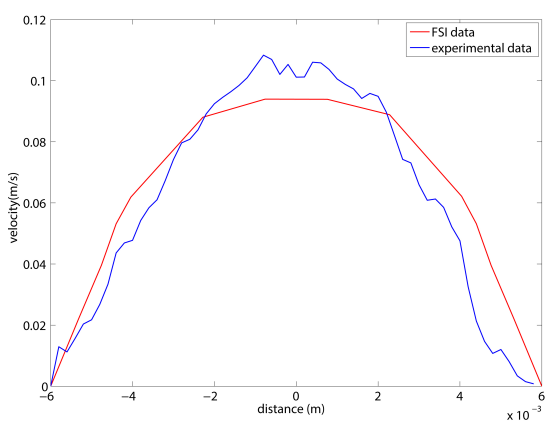

(a)

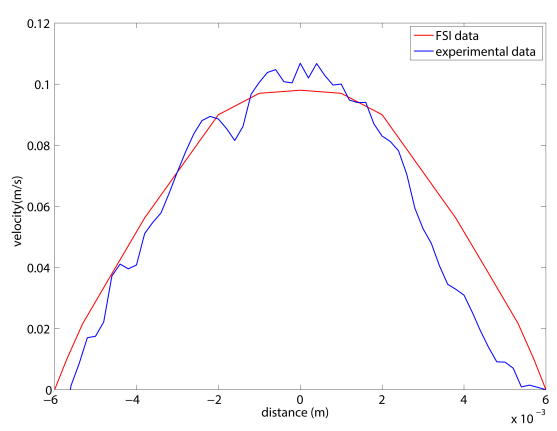

(b)

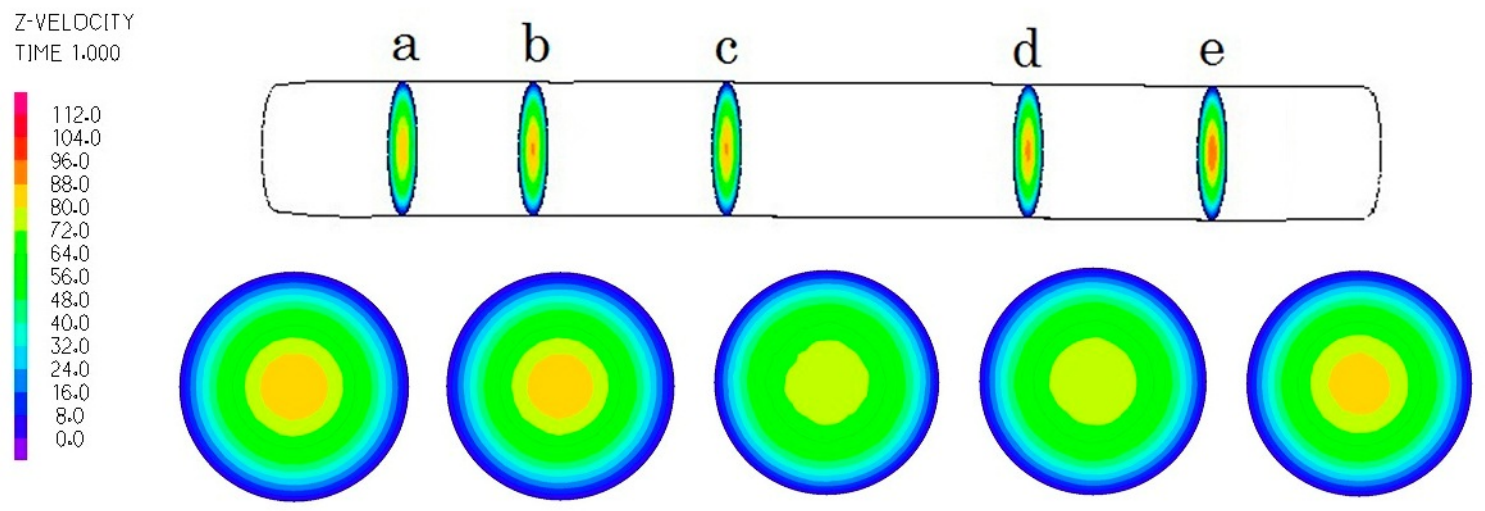

(c)

Fig. 2. Velocity profile for the simplified vena model a) at a point close to the inlet and b) at a point close to the outlet. c) velocity isocontours along the vessel axis 
Z-VELDCITY

TIME 1.000

112.0
104.0
96.0
88.0
80.0
72.0
64.0
56.0
48.0
40.0
32.0
24.0
16.0
8.0
0.0

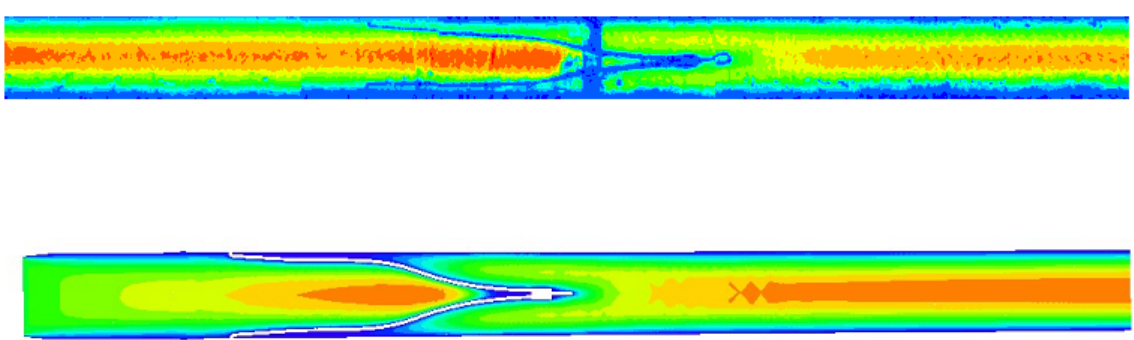

Fig. 3. Velocity profiles in the symmetry plane of the model containing the filter (top: FSI model, bottom; experimental model 


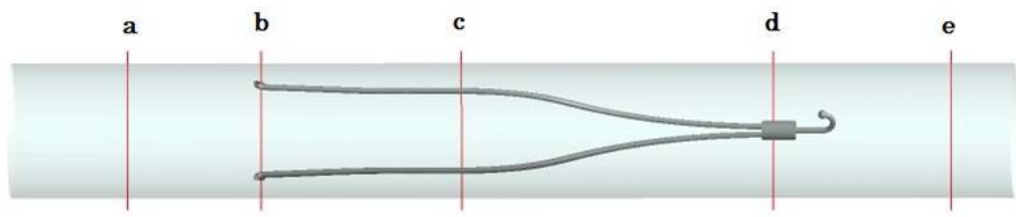

(a)

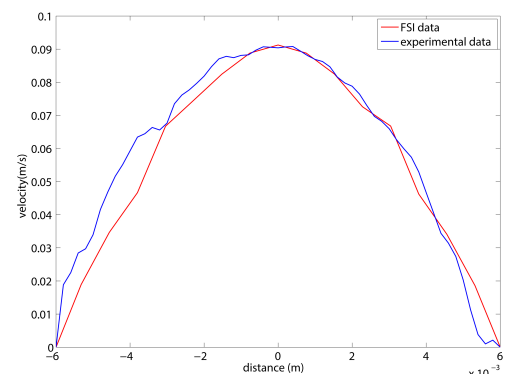

(b)

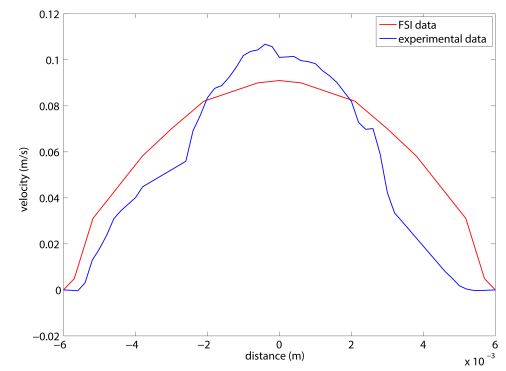

(d)

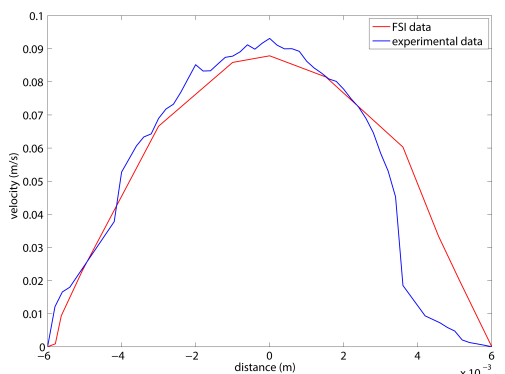

(c)

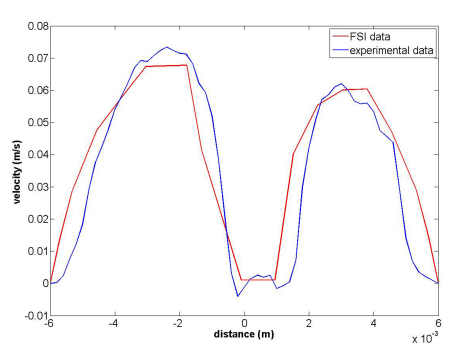

(e)

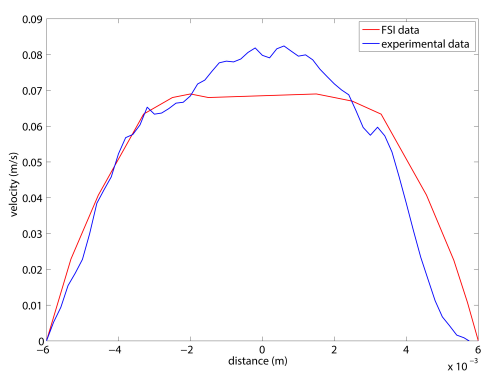

(f)

Fig. 4. Velocity profile for the filter model along lines a, b, c, d and e 


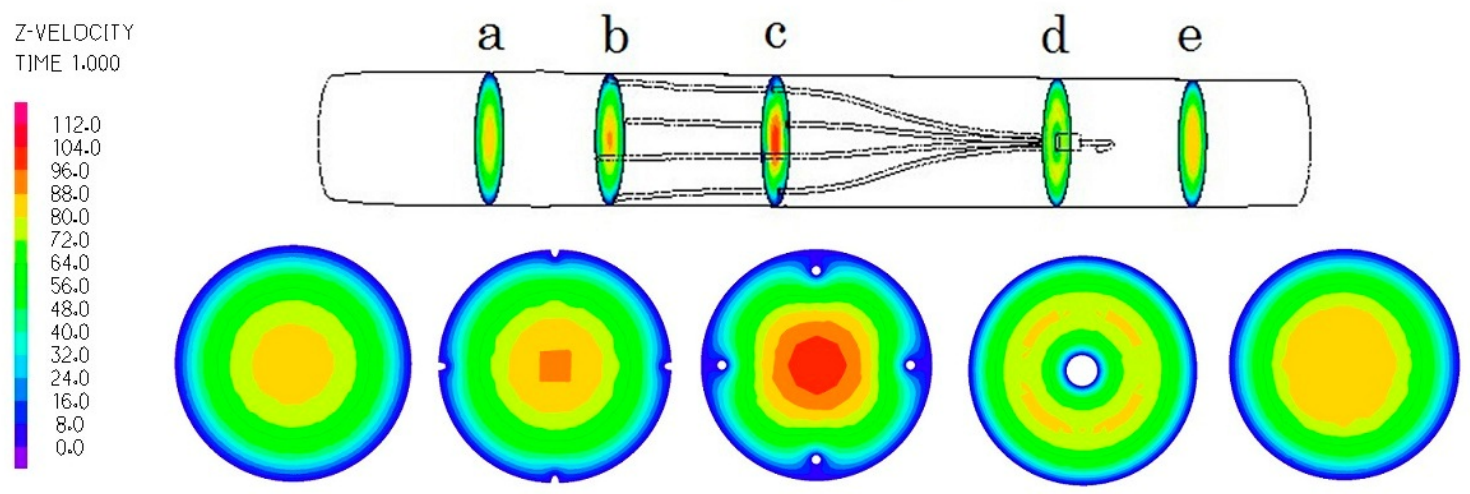

Fig. 5. Velocity contours on cross-sectional slices in the filter/vena model
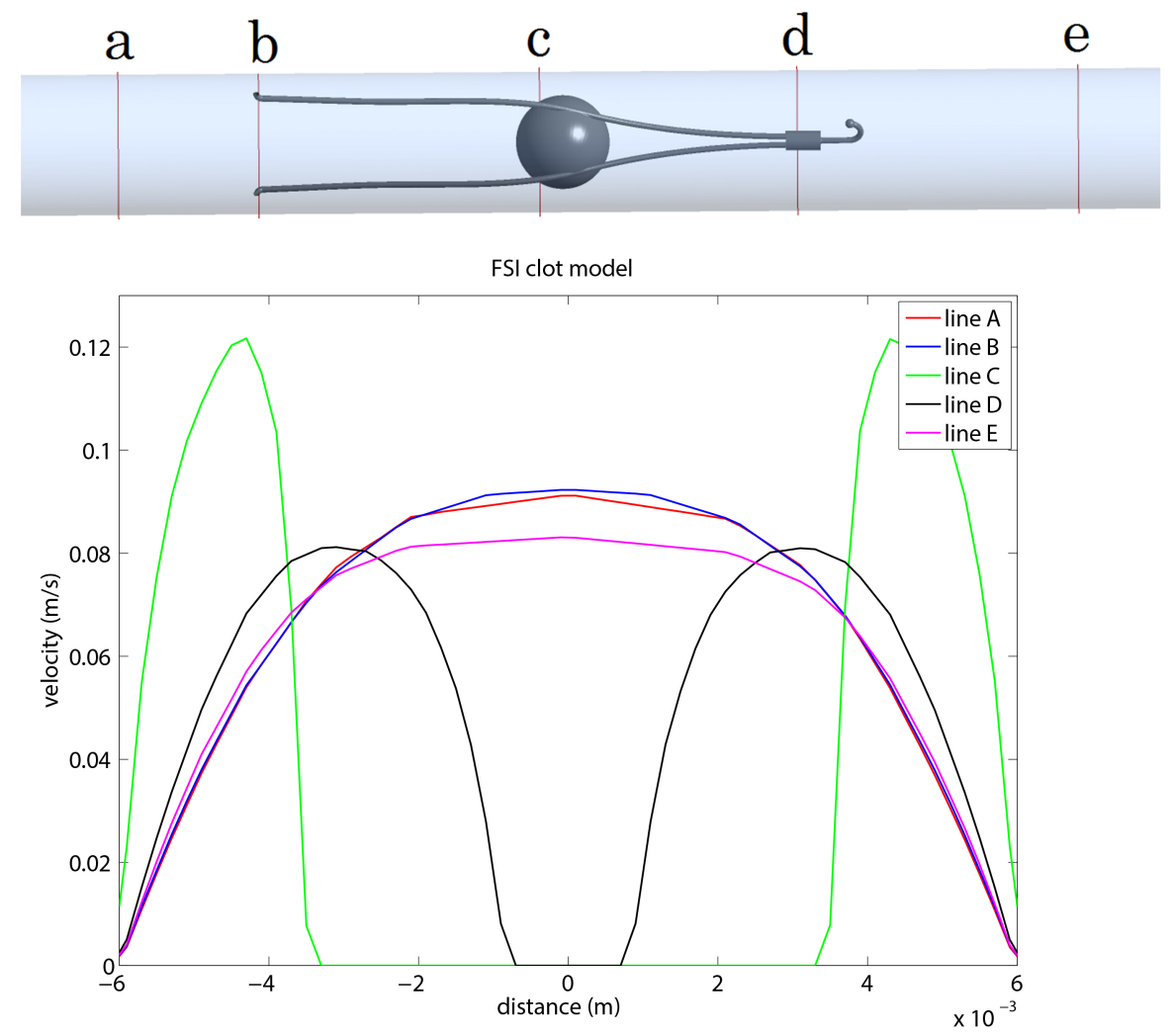

Fig. 6. Velocity contours on cross-sectional slices in the filter/vena model 

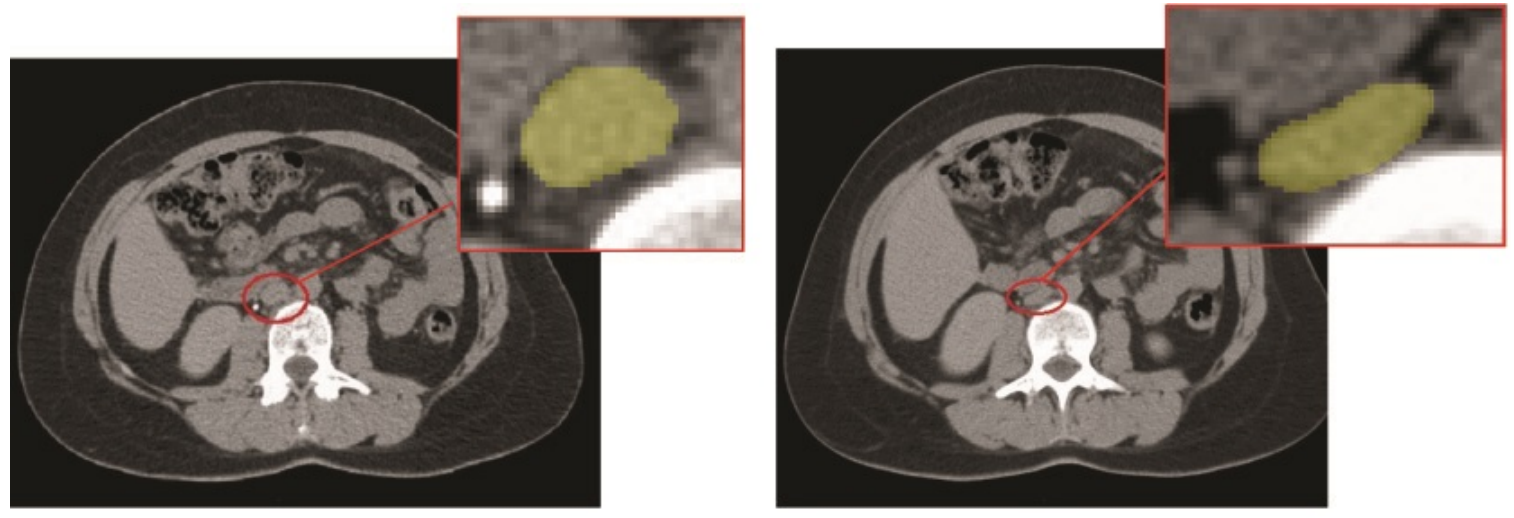

Fig. 7. Shape of the vena cava under normal and valsalva conditions

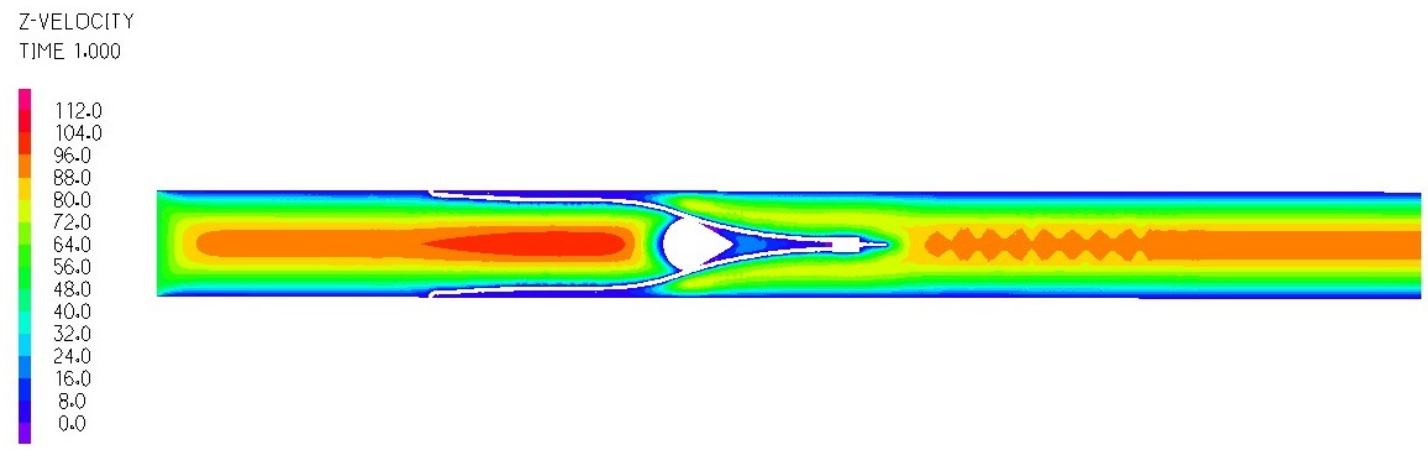

(a)

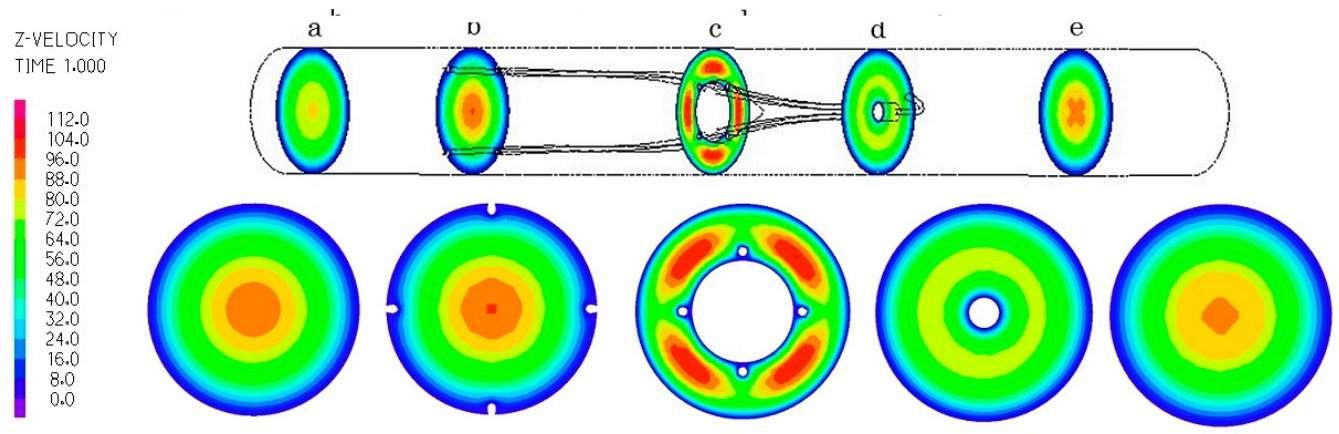

(b)

Fig. 8. Velocity profile for the simplified vena model a) at a point close to the inlet and b) at a point close to the outlet. c) velocity isocontours along the vessel axis 